\title{
Business Intelligence in Collaborative Networks
}

\section{Towards New Services for Moderation Management}

\author{
Heiko Thimm¹, Karsten Boye Rasmussen² \\ ${ }^{1}$ School of Engineering, Pforzheim University \\ ${ }^{2}$ Department Marketing and Management, \\ University of Southern Denmark, Odense
}

\section{Introduction}

Implementation of the concepts of networking and collaboration have been promoted as a successful approach when dealing with the present business challenges especially for small and medium sized enterprises (SMEs). As a result more and more collaborative networks (Camarinha-Matos and Afsarmanesh 2005, pp. 439452; Grandori and Soda 1995, pp. 183-214) are emerging in which companies come together to jointly act on the market. For given business opportunities this means that following a selection process a created subset of the network members forms a temporary virtual enterprise to transform the opportunity into profit which implies a set of collaborative business processes that are to be coordinated.

Collaborative networks must be supported by an appropriate IT infrastructure that offers suitable operational services in order to provide value for business partners. These computer-mediated services will include special information and decision support services for effective moderation of the collaboration within the network by a human moderator.

Moderation management in collaborative networks can be broadly described as the set of strategic and operative tasks that are carried out by a human moderator in order to meet the objectives of the network. As management tasks in single companies are supported by dedicated IT applications, also moderation management in collaborative networks requires from the IT infrastructure dedicated services. In our research framework we investigate especially the operative level of the decision making practice in collaborative networks with the goal to invent and experiment with new services for moderators and the network members.

We present two of such new services: 1) decision support of moderators, and 2) transparency support of the moderator and the network members. Both services support the concept of Business Intelligence (BI) in the sense that they are based 
upon complex data analyses performed on a comprehensive data repository. The processes, however, are not drawing upon the usual BI building blocks such as a data warehouse or a processor for Online Analytical Operations (OLAP).

Our decision support service enables moderators to effectively perform the selection decisions for the forming of virtual enterprises (Thimm et al. 2008). The transparency support service allows for an automated completion of the moderator's communication task. When a proposed virtual enterprise configuration alternative is chosen from the result list of the decision support service the moderator can instruct the transparency support service to generate and distribute the data rich decision explanations within the network.

In our article this first introductory section is followed by a section presenting a comprehensive investigation of the particular tasks of network moderators. The third section introduces our proposed services. Section four contains an evaluation of our decision explanations and describes some intended future work. Section five discusses related work while concluding remarks are given in section six.

\section{Investigated Moderation Management Tasks}

The decision making tasks of moderators of collaborative networks can include the configuration of specific subsets of the network members that form temporary alliances regarded as Virtual Enterprises (VE). The moderator is called to perform this task for every new business request for which a collaborative request handling within the network is demanded. Naturally, it is a goal of this task to configure a VE that precisely fits to the request. Therefore, the companies' profiles and competences, resource utilization states, and other company specific criteria as well as criteria that relate to the network as a whole are to be considered. For this reason, the configuration of a VE imposes to the moderator a complex multi-criteria decision problem (Thimm et al. 2008). To be considered in the context of this problem are both hard and soft selection criteria that relate to single network members. But also criteria that relate to the network as a whole need consideration. Not only is the number of selection criteria increasing with the size of the network. The number and complexity of the offered products and the number of interdependencies between the network members such as overlapping competences between companies are also growing fast. Furthermore, the criterions' relevance for the VE configuration are also changing over time as a result of market changes but also based upon changes in the relations between the network members.

Members of collaborative networks will feel uncomfortable if configuration decisions concerning VEs are not communicated according to the principles of robust decision downloading (Clampitt and Williams 2007). By this notion a mode of communication is conceptualized where the following information about decisions will be conveyed to those who have not been directly involved in the decision making process: 1) how and why the decision was made, 2) what alternatives were 
considered, 3) how the decision fits with the organizational mission, 4) how the decision impacts the organization and employees. The transparency and availability of these information items that explain VE configuration decisions will lead to greater decision acceptance. Furthermore, as research on organizational justice has shown robust decision downloading will lead to a number of advantages with respect to implications on individual employees. These advantages include a stronger support of and commitment to the organization, a higher identity with the organization, and an employee perception that the organization is well managed and headed in the right direction (Clampitt and Williams 2007). It is a hypothesis of our research that these findings are to a large extent valid when aggregated to decision making in collaborative networks, too. We assume that the members of such networks, i.e. companies of typically smaller and medium size, can be compared to the individual employees in the classical decision downloading context.

Robust decision downloading in collaborative networks is especially useful for decisions that influence the economic situations of the network members. This condition holds true for the configuration of VEs that in general imply a separation of the network members into two groups. On the one side there is the group of members that will benefit from the decision because they will be assigned to work on a business opportunity and, thus, experience or at least expect a revenue opportunity. On the other hand, there is the group of network members that cannot expect directly benefit from the decision because they are not selected to participate in the VE. The group of non-benefiters can be further divided into network members that for obvious reasons do not participate in the VE. For example, they might not offer any service or product needed for the fulfillment of the business request. However, the group of non-benefiters can also consist of companies that offer exactly the services and products needed and that have been considered for the VE but for other less obvious reasons have not been selected for the final VE. For example, they might have participated in many previous VEs or they might have been explicitly excluded as potential collaboration partners by other members that are definitely needed for the VE.

Communicating VE configuration decisions properly is especially demanded by the group of non-benefiters. In our view a proper communication mode will contribute to a broad acceptance for the configuration decision within the entire network (and not only by the group of benefiters). This will in the long run be beneficial for a pro-networking spirit and an open and trustful collaboration climate. Consider in this context that most often the network members will have some non-benefiter experience when we assume the facts that required competencies and configuration criteria for VEs will be different from business request to business request and that the network consists of a large number of rather specialized companies. 


\section{Overview of Proposed New Services}

Figure 1 contains a conceptual view of the proposed services showing the supported moderator activities (depicted as the pentagons on the top) and the utilized information bases (boxes at the bottom).

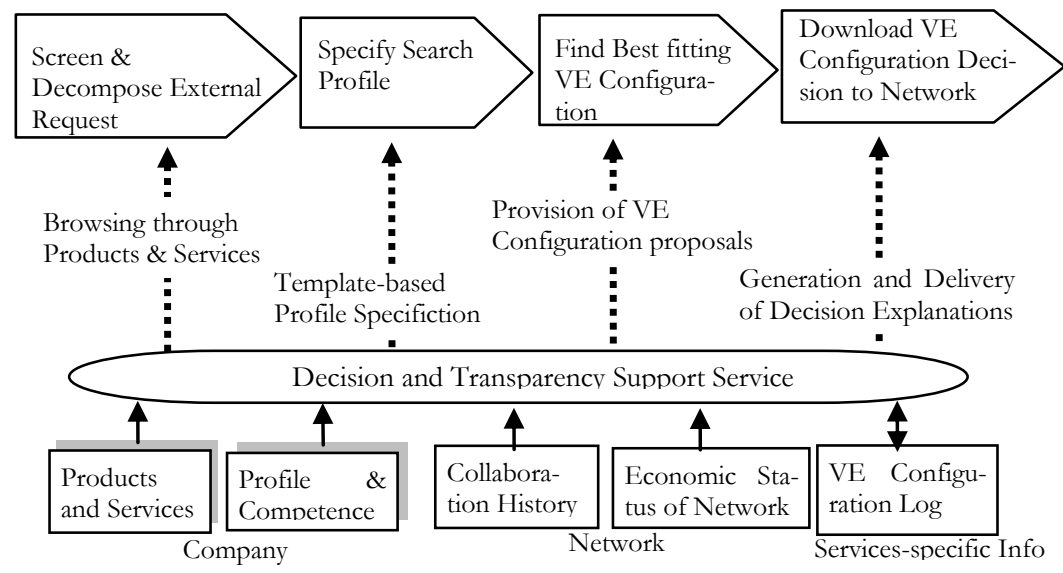

Figure 1: Conceptual view of proposed services

These information bases concern the product and service offerings of the network members and also their company profiles, competences, and collaboration preferences. The information base also stores the collaboration history of the company network, i.e. records describing previous VEs including information about the corresponding requests and the resulting collaboration experience. The information base also consists of the economic status of the company network in the form of the typical key performance indicators such as cash balance, order backlog, resource utilization, and inventory data. Furthermore, recorded moderator sessions are also stored within the information base.

\subsection{Decision Support Service (DSS)}

The creation of a new VE is performed in three steps. In the first step, the external business request is screened and decomposed into a set of corresponding categories of request handlers that are needed for the fulfilment of the request. In principle these categories refer to products and service offerings of the network that are usually supplied by multiple competing network members. The Decision Support Service (DSS) allows the moderator to browse through these categories and the associated companies as defined within the information base. Supported by this service the moderator can prepare for a suitable decomposition of the request into a corresponding set of request handlers.

In the second step, an initial search profile for the demanded VE is specified which states the set of needed kinds of request handlers and a set of criteria for 
selecting companies and evaluating possible VE alternatives. In order to allow for convenient specification of the search profile a corresponding interactive template is supplied to the moderator. The content of the template's selection boxes are dynamically queried from the information base. The predefined selection criteria of the template are divided into hard and soft selection criteria. Hard selection criteria consist of inclusion and exclusion constraints. It is possible through the definition of such constraints to include or exclude particular companies for the targeted VE. We refer to these criteria as collaboration constraints. Soft selection criteria are used for scoring single companies and VE configuration alternatives, respectively. We refer to these criteria as configuration criteria. At the current stage of our research we consider as criteria for scoring single companies the financial power, production/service quality, price level, and collaboration experience. The current set of criteria for scoring entire VE configuration alternatives includes the geographical proximity of the VE members, the current state of revenue distribution and workload distribution within the network. A numeric weight has to be assigned to each of these predefined criteria of the search profile template. In general, by prioritizing the different criteria through a corresponding assignment of weights the moderator may flexibly customize the scoring process of the DSS to address requirements. A more comprehensive and more formal specification of the different criteria can be found in (Thimm et al. 2008).

Once the search profile is fully defined it is submitted to the DSS. The service in turn generates valid VE alternatives that meet the hard selection constraints. Following that the alternatives are scored with respect to their goodness of fit to the soft selection constraints. In order to compute these scores a comprehensive data analysis of the information base is performed. In the third step, a ranked list of $\mathrm{VE}$ alternatives resulting from the scoring process is returned to the moderator for further evaluation. Either this will lead to another iteration starting with a modified search profile. Or it is decided for one of the proposed VE alternatives in the result list. The final decision is declared to the DSS where the decision and all preceding interactions between the moderator and the DSS are recorded for later analysis by the transparency support service. Moderators may through the DSS achieve what is generally regarded as "informed decision making" (Dave 1995, pp. 169-173). That is, by iterating over the above described three steps several times with different versions of search profiles the moderator can obtain deep insights into the decision problem space and explore corresponding VE alternatives.

\subsection{Transparency Support Service (TSS)}

In order to achieve a high level of acceptance for $\mathrm{VE}$ configuration decisions by the network members well prepared information are to be communicated in a proper mode of communication within the network. The Transparency Support Service (TSS) is intended to provide an effective means for a highly automated and efficient completion of this communication task. By the use of the TSS moderators 
can complete this decision downloading task with only a minimal time effort. Well prepared information regarded as decision explanations of the rationales behind the VE configuration decisions will be automatically generated and distributed in the network. An automated generation of the decision explanations is enabled by a machine processible representation of the final decision and the path from the initial search profile over all the completed iterations up to the final choice.

The decision explanations are generated in three steps. A comprehensive data analysis of the information base is performed in each of these steps. Firstly, the relevant decision justification information is derived from the individual decisionspecific criteria, global policies, and strategies defined for the network, and corresponding information about the current global status of the network. In a second step this information is augmented by further context specific background information to make it easier for users to perform a decision diagnosis and to gain understanding about the decision justification. In a third step the TSS generates decision explanations which consist of meaningful quantitative data related to the decision and which are partially individualized on a per network member basis. Examples for these decision explanations are given in the next section in which we also discuss the limitations of the current state of our research and our future plans, too.

The decision explanations are stored in a dedicated repository. For their distribution within the network several alternative distribution mechanisms can be considered. At the current state of our research the distribution is performed similar to email newsletters. The emails received by all network members contain the same general announcement about the forming of a new VE within the network and the corresponding business request. This information is complemented by individualized access information to receiver-specific decision explanations in the form of an. By this means the network members can conveniently fetch their own decision explanations at any given time from the repository over a secured connection.

\section{Decision Explanations - Evaluation and Outlook}

At the present state three types of decision explanations are considered. We describe these types by showing a concrete example that is based on a simplified application scenario. For this scenario we suppose a fictive company network specialized on the production of passenger seats for planes, ships, trains, and busses. We assume that this network has received a request for quotation from a shipyard asking for an offer for 400 passenger seats with an integrated infotainment system. From a corresponding process description for the production of the requested seats the moderator can deduce that the following set of activities is needed for the order fulfilment: (1) production of metal seat frames, (2) production of seat upholsteries, (3) production of circuit systems, (4) production of monitors, (5) production of harnesses, (6) final assembly of seats. From the set 
of request handler categories available in the network the moderator will choose six categories that fit to these activities. A corresponding selection box for this task is part of the DSS's search profile template. The further specifications defined by the moderator within the search profile are in the example (Table 1) as follows:

- The network member with the company name "SUM Microelectronics Ltd." has to be included in the VE. This include constraint can arise from a corresponding inquiry of the requester.

- The network member with company name "Iron Experts Ltd." is to be excluded in the VE which is expressed through a corresponding exclude constraint.

- The configuration process for generating scored VE alternatives has to consider two company-related configuration criteria. As one criterion "Collaboration Experience: 0.6" is selected and as further criterion "Financial Power: 1.0" is selected. This choice means that the scoring of individual members is performed with respect to these two criteria and their assigned weights, respectively.

- The configuration process needs also to reflect the network-related configuration criteria "Equally Balanced Revenue: 0.8" and "Equally Balanced Workload: 1.0". The scoring of alternative VE configurations will consider these two criteria accordingly.

The initial search profile as described above is processed by the DSS which will deliver a resulting list of scored VE alternatives. Let us assume that the moderator will not perform further iterations and directly decide for the top scoring VE alternative. This decision is in turn downloaded to the network by the TSS which implies that corresponding decision explanations are generated and delivered to the network members.

Three examples of different types of decision explanations that the TSS will generate for the above described scenario are presented in the following. These types of decision explanations are referred to by the general notion of view to reflect that the explanations are based on data analyses from different points of view.

The Search Profile View presents the search profile as specified by the moderator. It is divided into three parts as shown in the example of Table 1 that reflects the above described application scenario. The first part contains the set of request handler categories. The second part shows the collaboration constraints. Note that multiple include and exclude collaboration constraints, respectively, can be given in the Search Profile View in general. However, in our sample scenario only one concrete constraint of each type is given. The third part of the view presents the configuration criteria. 
Table 1: Search Profile View of sample application.

\begin{tabular}{|c|c|c|c|c|c|c|}
\hline \multicolumn{7}{|c|}{ 1. Request Handlers } \\
\hline $\begin{array}{l}\text { Deman- } \\
\text { ded set } \\
\text { of } \\
\text { request } \\
\text { handlers }\end{array}$ & $\begin{array}{l}\text { Metal } \\
\text { Constr. } \\
\text { Prod. }\end{array}$ & $\begin{array}{l}\text { Up- } \\
\text { holstery } \\
\text { Prod. }\end{array}$ & $\begin{array}{l}\text { Elec. } \\
\text { Parts } \\
\text { Prod. }\end{array}$ & $\begin{array}{l}\text { Moni- } \\
\text { tor } \\
\text { Prod. }\end{array}$ & $\begin{array}{l}\text { Har- } \\
\text { ness } \\
\text { Prod. }\end{array}$ & $\begin{array}{l}\text { As- } \\
\text { semb } \\
\text { by } \\
\text { Spec. }\end{array}$ \\
\hline \multicolumn{7}{|c|}{ 2. Collaboration Constraints } \\
\hline \multicolumn{3}{|c|}{$\begin{array}{l}\text { include collaboration } \\
\text { constraints }\end{array}$} & \multicolumn{4}{|c|}{ SUM Microelectronics Ltd. } \\
\hline
\end{tabular}

\begin{tabular}{|l|l|l|}
\hline $\begin{array}{l}\text { exclude collaboration } \\
\text { constraints }\end{array}$ & \multicolumn{2}{|c|}{ Iron Experts Ltd. } \\
\hline \multicolumn{2}{|c|}{ 3. } & \multicolumn{1}{|c|}{ Configuration Criteria } \\
\hline $\begin{array}{l}\text { Company-related } \\
\text { configuration } \\
\text { criteria }\end{array}$ & $\begin{array}{l}\text { Collaboration } \\
\text { Experience: } 0.6\end{array}$ & $\begin{array}{l}\text { Financial } \\
\text { Power: } 1.0\end{array}$ \\
\hline $\begin{array}{l}\text { Network-related } \\
\text { configuration } \\
\text { criteria }\end{array}$ & $\begin{array}{l}\text { Equally } \\
\text { Balanced } \\
\text { Revenue: } 0.8\end{array}$ & $\begin{array}{l}\text { Equally } \\
\text { Balanced } \\
\text { Workload: } 1.0\end{array}$ \\
\hline
\end{tabular}

The Search Result and Criteria Evaluation View is intended to clarify to the company representatives the reasons for the final decision. In particular arguments are provided as to why their company is a part of the VE or, in the opposite case, why their company has not been selected. As shown in Table 2 four sets of clarification information are given. The first set contains the moderator's final decision by showing the companies that participate within the chosen $\mathrm{VE}$ as request handlers. The second set shows the total score of the chosen VE and also the minimum, mean, and maximum score of all considered VE alternatives. The third set consists of VE-related scoring data. For each considered network-related scoring criterion the score of the chosen $\mathrm{VE}$ alternative is given and contrasted with the corresponding minimum, mean, and maximum scores of the set of all considered $\mathrm{VE}$ alternatives. The fourth set of the view consists of company-related scoring data which is individualized on a per company basis. For example, the fourth part of the view in Table 2 is individualized for the network member "SUM Microelectronics Ltd." and, henceforth, presents the scoring results of that company with respect to the company-related scoring criteria.

Table 2: Search Result \& Criteria Evaluation View for SUM Microelectronics Ltd.

\begin{tabular}{|l|l|l|l|l|l|l|}
\hline \multicolumn{5}{|c|}{ 1. Request Handlers } \\
\hline $\begin{array}{l}\text { Chosen set } \\
\text { of request } \\
\text { handlers }\end{array}$ & $\begin{array}{l}\text { SUM Micro- } \\
\text { elec. Ltd. }\end{array}$ & $\begin{array}{l}p . \\
2\end{array}$ & $\begin{array}{l}p . \\
3\end{array}$ & $\begin{array}{l}\text { T. } \\
4\end{array}$ & $p .5$ & $p .6$ \\
\hline \multicolumn{3}{|c|}{\begin{tabular}{l} 
Total Scores \\
\hline Type of VE score
\end{tabular}} & $\begin{array}{l}\text { VE-score } \\
\text { of chosen } \\
\text { VE } \\
\text { alternative }\end{array}$ & $\begin{array}{l}\text { VE-scores found } \\
\text { among set of all } \\
\text { considered VE } \\
\text { alternatives }\end{array}$ \\
\hline Total VE score & $\begin{array}{l}\text { Min.: 78 } \\
\text { Mean: 126 } \\
\text { Max.: 199 }\end{array}$ \\
\hline 3. VE-Related Scoring Data \\
\hline Scoring criterion & $\begin{array}{l}\text { VE-score } \\
\text { of chosen } \\
\text { VE } \\
\text { alternative }\end{array}$ & $\begin{array}{l}\text { VE-scores found } \\
\text { among set of all } \\
\text { considered VE } \\
\text { alternatives }\end{array}$ \\
\hline
\end{tabular}

\begin{tabular}{|c|c|c|}
\hline $\begin{array}{l}\text { Equally Balanced } \\
\text { Revenue: } 0.8\end{array}$ & 89 & $\begin{array}{l}\text { Min.: } 67 \\
\text { Mean: } 72 \\
\text { Max.: } 91\end{array}$ \\
\hline $\begin{array}{l}\text { Equally Balanced } \\
\text { Workload: } 1.0\end{array}$ & 110 & $\begin{array}{l}\text { Min.: } 56 \\
\text { Mean: } 83 \\
\text { Max.: } 110\end{array}$ \\
\hline \multicolumn{3}{|c|}{$\begin{array}{l}\text { 4. Company-Related Scoring Data for SUM } \\
\text { Microelectronics Ltd. }\end{array}$} \\
\hline $\begin{array}{l}\text { Scoring } \\
\text { criterion }\end{array}$ & $\begin{array}{l}\text { Company- } \\
\text { score of own } \\
\text { company }\end{array}$ & $\begin{array}{l}\text { Company-scores } \\
\text { found among set } \\
\text { of all considered } \\
\text { alternatives }\end{array}$ \\
\hline $\begin{array}{l}\text { Collaboration } \\
\text { Experience: } \\
0.6\end{array}$ & 73 & $\begin{array}{l}\text { Min.: } 58 \\
\text { Mean: } 62 \\
\text { Max.: } 73\end{array}$ \\
\hline $\begin{array}{l}\text { Financial } \\
\text { Power: } 1.0\end{array}$ & 120 & $\begin{array}{l}\text { Min.:49 } \\
\text { Mean: } 72 \\
\text { Max.:120 }\end{array}$ \\
\hline
\end{tabular}


In order to allow for a better interpretation of the scoring information the corresponding minimum, mean, and maximum scores concerning the set of all considered companies are given in this fourth part of the view, too. A network member may use this benchmarking information for a strategic alignment of the company to the specific properties and strategy of the collaborative network. For example, this alignment may lead to particular long term investments in production facilities and employee skills.

The Decision Impact View is based on a projection of quantitative data into the future. This view clarifies the anticipated consequences of the given $\mathrm{VE}$ configuration decision for both the network as a whole as well as for single members of the network. In order to allow insights into the possible decision impact on the network qualitative and quantitative indicators are described in terms of their current status and their assumed future development. The values in the column "future development" describe future states of the indicators as they will result when the business request is handled by the chosen VE. For some of these indicators the corresponding numbers for the entire network and also for the own company are given. For example, in the view of Table 3 the data contained in the first part refers to the entire network whereas the data of the second part is company specific and refers to "SUM Microelectronics Ltd."

Table 3: Sample Decision Impact View for SUM Microelectronics.

\begin{tabular}{|c|c|c|}
\hline \multicolumn{3}{|c|}{ 1. Decision Impact on Entire Network } \\
\hline Indicator & current status & future development \\
\hline Revenue & $\begin{array}{l}\text { network: } 10.500200 \\
\text { per member: } 620000\end{array}$ & $\begin{array}{l}\text { network: } 11.800000 \\
\text { per member: } 710000\end{array}$ \\
\hline $\begin{array}{l}\text { Revenue } \\
\text { distribution }\end{array}$ & unbalanced & slightly unbalanced \\
\hline Utilization & low & $\begin{array}{l}\text { Short term: normal } \\
\text { Medium term: normal } \\
\text { Long term: - }\end{array}$ \\
\hline Inventory & normal & $\begin{array}{l}\text { Short term: low } \\
\text { Medium term: normal } \\
\text { Long term: - }\end{array}$ \\
\hline VE size & $\begin{array}{l}\text { Min.: } 4 \\
\text { Mean: } 12 \\
\text { Max.: } 24\end{array}$ & $\begin{array}{l}\text { Min.: } 4 \\
\text { Mean: } 10 \\
\text { Max.: } 24\end{array}$ \\
\hline VE value & $\begin{array}{l}\text { Min.: } 80000 \\
\text { Mean: } 6.500000 \\
\text { Max.: } 15.000000\end{array}$ & $\begin{array}{l}\text { Min.: } 80000 \\
\text { Mean: } 5.665000 \\
\text { Max.: } 15.000000\end{array}$ \\
\hline $\begin{array}{l}\text { Waiting } \\
\text { Time }\end{array}$ & $\begin{array}{l}\text { Min.: } 4 \\
\text { Mean: } 8 \\
\text { Max.: } 15\end{array}$ & $\begin{array}{l}\text { Min.: } 4 \\
\text { Mean: } 6 \\
\text { Max.: } 15\end{array}$ \\
\hline
\end{tabular}

\begin{tabular}{|c|c|c|}
\hline & \multicolumn{2}{|c|}{$\begin{array}{l}\text { 2. Decision Impact on SUM } \\
\text { Microelectronics Ltd. }\end{array}$} \\
\hline Indicator & \begin{tabular}{|l|} 
current status \\
\end{tabular} & future development \\
\hline Revenue & 840000 & 1.251000 \\
\hline Utilization & low & $\begin{array}{l}\text { Short term: high } \\
\text { Medium term: high } \\
\text { Long term: - }\end{array}$ \\
\hline Inventory & normal & $\begin{array}{l}\text { Short term: low } \\
\text { Medium term: low } \\
\text { Long term: - }\end{array}$ \\
\hline VE size & $\begin{array}{l}\text { Min.: } 6 \\
\text { Mean: } 8 \\
\text { Max.: } 15\end{array}$ & $\begin{array}{l}\text { Min.: } 6 \\
\text { Mean: } 8 \\
\text { Max.: } 15\end{array}$ \\
\hline VE value & $\begin{array}{l}\text { Min.: } 80000 \\
\text { Mean: } 3.700000 \\
\text { Max.: } 8.200000\end{array}$ & $\begin{array}{l}\text { Min.: } 80000 \\
\text { Mean: } 2.400000 \\
\text { Max.: } 8.200000\end{array}$ \\
\hline $\begin{array}{l}\text { Waiting } \\
\text { Time }\end{array}$ & $\begin{array}{l}\text { Min.: } 6 \\
\text { Mean: } 8 \\
\text { Max.: } 15\end{array}$ & $\begin{array}{l}\text { Min.: } 6 \\
\text { Mean: } 8 \\
\text { Max.: } 15\end{array}$ \\
\hline
\end{tabular}


At the current state of our research we take only a limited set of indicators into account. As economic indicators considered are:

- Revenue: Accumulated revenue number for the current business year as obtained through the network. The number for the entire network and the mean number for the members are given.

- Revenue distribution: Description of revenue distribution within the network in the form of values on an ordinal scale that ranges from unbalanced, slightly unbalanced, and balanced.

- Utilization: Degree of utilization of the resources given in the form of values of an ordinal scale that ranges from low, normal, up to high. The description of the future development is based on a separation into short term, medium term, and long term development of the utilization.

- Inventory: Description of amount of material on stock stated in the form of values of an ordinal scale that ranges from low, normal, up to high. Also for this indicator the future development is separated into corresponding values for short term, medium term, and long term.

Apart from the economic indicators other indicators more related to collaboration are also considered. In general, these collaboration-specific indicators are considered in two variants: one referring to the entire network and the other one referring to the own company. For each of these indicators three numbers are given that provide the minimum, mean, and maximum value. The collaboration-specific indicators are as follows:

- VE size: Size of VE in terms of number of participating companies. The company specific number in the second part of the view refers to VEs that the own company has participated in.

- VE value: Overall monetary business value of the referring business request. The numbers given for the own company refer to the VEs that the own company has participated in.

- Waiting time: Time span in days between end date of the corresponding prior $\mathrm{VE}$ and start date of the next VE where the companies are to participate in. Like above the company specific number refers to VEs with participation by the own company and not all the VEs.

Several limitations of our current research results have to be considered. First of all the decision views are limited to a pure tabular presentation of information. Understanding the full meaning of their content can require too much time for users. In our future work we intend to make use of advanced visualization and layout techniques to overcome this restriction. Moreover, the content of views is focused on that particular VE alternative that has been selected by the moderator. The other VE alternatives considered by the DSS with lower scores (or even higher scores depending on the moderator's choice) are not explicitly shown in the views. 
These other VE alternatives are only reflected implicitly in the given VE-scores that refer to the set of all considered VE alternatives. However, given the goal of decision transparency we will extend our solution by corresponding information in the future. A possibility that we will investigate in this context is to evolve the current views to what can be regarded as active or online views which are generated on request. This concept of active view will allow the users to select a VE alternative considered by the DSS and through the TSS to obtain dynamically generated decision views with information about the selected VE. In the context of this enhancement we will investigate to place the TSS on top of a Business Intelligence (BI) infrastructure with corresponding multi-dimensional data cubes and an Online Analytical Processor (OLAP) for OLAP operations such as slice-and-dice and drilling down aggregated data across a multi-level aggregation hierarchy. However, since an aggregation of data in our context would span only a very limited number of aggregation levels, we so far assume that the information needs of the network members can be sufficiently managed through the offline decision views as presented above. Using such a full blown BI approach can also lead to the problem that the users of the network member companies who are likely to use the service on a casual basis can be over-challenged by the BI complexity which implies the aforementioned focus on user-friendly graphics.

It is another weakness of our approach that the current set of decision views is specialized on the information needs of network members. The information needs of network moderators are however much more sophisticated and, therefore, only partially covered by our current decision views. It is our plan to first obtain a better understanding of the concrete information demand of network moderators by conducting an empirical study. In this study our prototype will serve as vehicle to demonstrate the current status of our research and the study will also elicit new forms of decision views required by moderators.

\section{$5 \quad$ Related Work}

Business processes in collaborative networks including moderation management processes have been the target of several other research projects such as ECOLEAD (Camarinha-Matos and Afsarmanesh 2005, pp. 439-452). However, these studies have not particularly addressed moderation management processes where a network moderator can benefit from services that are based on BI technology. The same holds true for the communication of decisions within such networks and the impact on the network members in terms of trust and acceptance of the moderator. Noran (2009, pp. 4813-4832) in a recent article proposed a framework for a decision support system that can help managers and enterprise architects to discover/update the main activities and aspects that need to be modeled for various enterprise task types, with special emphasis on the creation of VEs. A system referred to as "decision platform" for collaborative networks has been proposed by 
Carlsson (2002, pp. 185-221). The design of this system is based on a multi-agent approach combining hyperknowledge and the use of multiple software agents with the latter being built on fuzzy logic and approximate reasoning. An interesting approach to compute trust estimates as selection criteria for VEs has been introduced by Lavrac et al. (2007, pp. 429-437). These trust estimates are very similar to the company specific scores used in our DSS. Complex data analysis techniques and a hierarchical multi-attribute decision-support approach are the foundation for the computation of these trust estimates.

\section{Conclusions}

In this article we introduced two novel services for collaborative networks that provide BI capabilities to moderators as well as to the member companies of collaborative networks. The decision and transparency support services will through their BI capabilities ease decision making for moderators and lead to better and less subjective decisions regarding the configuration of VEs.

\section{References}

Carlsson C (2002) Decision Support in Virtual Organizations: The Case for MultiAgent Support. Group Decision and Negotiation (11), Kluwer Academic Publishers:185-221.

Camarinha-Matos LM, Afsarmanesh H (2005) Collaborative networks: a new scientific discipline. Journal of Intelligent Manufacturing (16):439-452.

Clampitt P, Williams M (2007) Decision Downloading. MIT Sloan Management Review (Jan 1).

Dave G (1995) Informed decision making. Journal of Information Science 51(1):169-173.

Grandori A, Soda G (1995) Inter-firm Networks: Antecedents, Mechanisms and Forms. Organization Studies 16(2):183-214.

Lavrac N, Ljubic P, Urbancic T (2007) Trust Modeling for Networked Organizations Using Reputation and Collaboration Estimates. IEEE Transactions on Systems, Man, and Cybernetics 37(3): 429-439

Noran O (2009) A Decision Support Framework for Collaborative Networks. International Journal of Production Research 47(17):4813-4832

Thimm H, Thimm K, Rasmussen KB (2008) Supporting Moderators of Company Networks by an Optimization Service for Orchestration. In: Proceedings 16th European Conf. Information Systems (ECIS 2008), Galway, Ireland. 